（3）午面上材料的分布，能够分辩的距离为 0.5 $\mathrm{mm}$ 或更小。

（4）能达到的材料的深度或厚度在 $10 \mathrm{mg} / \mathrm{cm}^{2}$ 的 数量級。对于气体，这厚度要看它的密度或气压而定。 就固体說, 它相当于 $0.03 \mathrm{~mm}$ 的鋁屏或碳層。在适当 的情况，材料深度上的变化能分辨到全深的 $5 \%$ 。利 用能量較高的带电重粒子可以达到更深的地方。

（5）所用村料可很少, 相当于微量或超微量的范 固。

(6) 反应是瞬时的。

（7）材料在时間上的变化，在特定的情况可以分 辨到 1 秒鐘以內。1 分鐘是容易做到的。因此可能分 析本身在运动中的物質。

（8）材料的化学組成可有多种。混合物也可。反 应产額主要看被檢驗的同位素的濃度。

由上迅的特性可見这方法包含一些現在其他方法 （包括筫譜分析、放射性同位素方法、中子或带电 粒 子照射活化法[1]) 所未能做到的方面。它的缺点和限 制是由所需的設备、所能利用的核反应和材料中混入 某些杂筫的情况决定的。

这里举几个初步的可供探討的例子。

(1) 在分离氮的同位素- $\mathrm{N}^{14}$ 和 $\mathrm{N}^{15}$ 一的过 程中, 产物可用 1-2 Mev 的質子冲击,利用下列反应 产生的 $\gamma$ 射綵測定它的同位素成份:

$$
\begin{aligned}
\mathrm{N}^{14}+p & \rightarrow \mathrm{O}^{15}+\gamma \\
\mathrm{N}^{15}+p & \longrightarrow \mathrm{C}^{12 *}+a \\
\mathrm{C}^{12 *} & \rightarrow \mathrm{C}^{12}+\gamma(4.43 \mathrm{Mev})^{[2]}
\end{aligned}
$$

用現有的測量方法， $\mathrm{N}^{14}$ 的成份可低于 $5 \%$ ，而 $\mathrm{N}^{15}$ 的 成份則可低于 $0.03 \%$ 。其他 $\mathrm{Li}, \mathrm{B}, \mathrm{C}$ 等也可作类似 的分析。在用电磁分离或表面扩散的分离方法时，产 物所附着的表面即可值接作为靶子。这方法的准确度 不及筫譜分析，但有它的便利之处。

（2）鋁 (Al ${ }^{27}$ ) 既無其他稳定同位素文無寿命够 长的放射性同位素。在同位素应用上可用的激活反应 $\mathrm{Al}^{27}(\boldsymbol{n}, \boldsymbol{a}) \mathrm{Na}^{24}$ 能作較大量的分析而不易作微細結 构的分析。但在 $\mathrm{Al}^{27}(p, \gamma) \mathrm{Si}^{28}$ 反应中，有高产額的 $\gamma$ 射，及寬度小于几个 $\mathrm{Kev}$ ，因此可当作汥有宽度 的单的共振峰[3]。变更冲古質子的能量, 由所得的 $\boldsymbol{\gamma}$ 射可以測量鋁的薄居的厚度和梁度分布。例如在 牛导体上利用溜射或扩散法加上薄首的鋁的导体时， 即可用这方法研究。具有足够能量的質子可先穿过一 居其他物再到达內部的鋁尾。

碳的薄居或梁度分布也可用类似方法。这在金㞗 或有机物的研究上可能有用。 $C^{12}(p, \gamma) N^{13}$ 在 0.46 $\mathrm{Mev}$ 处, $\mathrm{C}^{13}(p, \gamma) \mathrm{N}^{14}$ 在1.76 Mev 处都有强共振峰。 此外，这方法可能应用的問題，如 C，B，F 等
在金屬、矿石或其他切片或表面上的牛微細結构，得 量的存在; 有关扩散、吸附、固体溶解等表面現象;

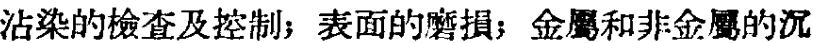
淀、沉积等过程; 混合的过程; 混合物的分析; 微柆 的分析; 在 $\gamma$ 射䋊及 $\beta$ 射厚度計所能应用的氾圍以 外的金屬或非金屬的薄居、細綫等的測量和控制等， 都是值得探討的。

李整武叶銘汉 (中国科学院物理研究所)

1957 年 4 月 12 日

[1] 例如 А. Н. Бродский, Химия Изотопов (Изд. АН

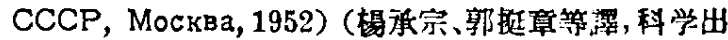
版社, 1956); Nucleonics, 14, No.5 (1956).

[2] F. Ajzenberg and T. Lauritsen, Revs. Mod. Phys., 27, 77 (1955).

[3] K. J. Broström, T. Huus and R. Tangen, Phys. Rev., 71, 661 (1947), P. M. Endt and J. C. Kluyver, Revs. Mod. Phys., 26, 95 (1954).

\section{光譜分析用的一种新的 双电弧电路}

“双电崛”[1]是一种簡伯的利用分餾来提高矿物試 样中易挥發元素光諧分析灵敏度的方法。自从它由 Shaw 等提出以后，已有好几简沋及应用双电弧的論 文發表 ${ }^{[2-5]}$ 。从电学上看，这些双电硍不过是两个电 联着的普通速緢直流或交流电弧，它們有着如下的主 要缺点:（1）激發电弧和加热电弧是电联的，在电学 上可以直接互相影响。(2) 激电张是个連緮电弧, 再現性差; “激带温度”不高, 不利于“难激荻元素”的 分析。这些缺点都使得过去的双电弧不能給出較好的 再現性 (Шиллинг[5] 用交流双电弧得到士17\% 的均力 偏差，其余的作者 ${ }^{[2-4]}$ 用直流时均方偏差达士 $30 \%$ ), 井使双电弧無法用来分析少量的 “难激發元素” 如㐫 素、硫等非金盛元素。

我們最近設計了一种双电崛电路, 它在很大的程 度上保存了前迌双电弧的优点而又免除了它們的缺 点。这电路的主要特点是:

(1) 加热的激电张在电学上是抹联的, 两个电 弧可以分别加以控制，互不影响。

（2）激电张采用高頻引燃、低电压电容放电， 稳定性高, 激放电可以由普通的引燃交流电弧（活 化交流电张）一直改变到“激犦温度”很高的强流低压 电花, 包括两者之間的所有过渡型或混合型的放电在 內。这样，电路就可以用来分析所有的易揮弗元素, 包括易揮洺而又难激的元素在內，相可以指望它 能給出較好的再現性。 


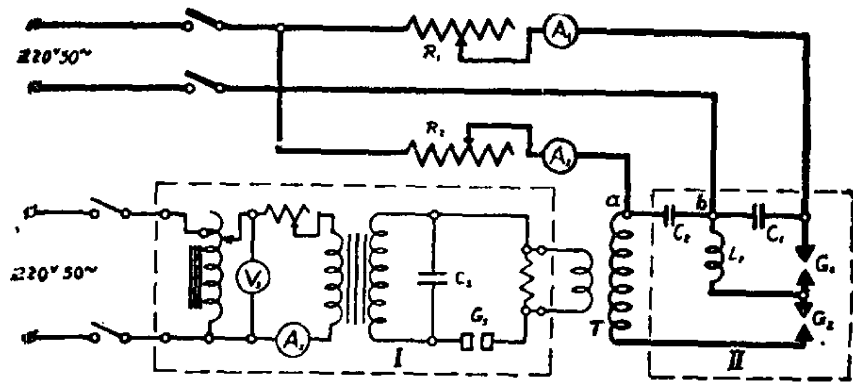

圆 1 双论础基本纨路简圆

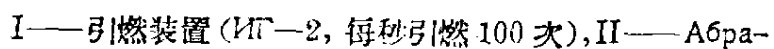
мсон 附加中路, T--Tesla 变压器, $C_{1}-L_{1}-G_{1}-$ 激發放电的论容放电迥路, $\mathrm{G}_{1}$ 一激發电讪, $\mathrm{G}_{2}$ 一加 热允弧， $\mathrm{C}_{2}$ 一一隔淔电谷。粗綫表示双近弧强流部分

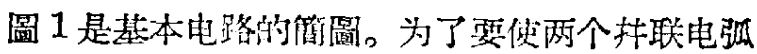
能同时引燃，我們乐用了 Абрамсон[6]的"付加电路，抹 加以修改, 使它合乎我們的裂求。乎們目前使用 $U \Gamma-2$ 型电花發生器作为设燃装置, 显然, 利用其他类型的

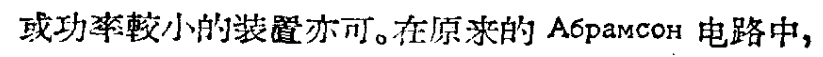
$c_{2}$ 只是一个輔助电花隲, 現在在 $a 、 b$ 两点間接入低 EE电隐, 抖去掉一个隔㨁电容, $G_{2}$ 也就成为一个功 焏可达几百正特以上的加热电弧了。除此之外, 电路 虳宁燃作用原理和 Абрамсон 所迅的一样。

改变电路中的 $R_{2}$ 則可改变加热电弧的电流, 而 激發条件不变。改变 $R_{1} 、 C_{1} 、 L_{1}$ 及 $G_{1}$ 則可改变激發 绦件，而不影响加热电㼋。

目的这电路还有这禁的缺点, 即在某些条件下

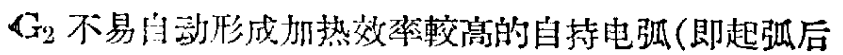
不必再引㦓也能維持不灭的电弧)。但是只要在通电后

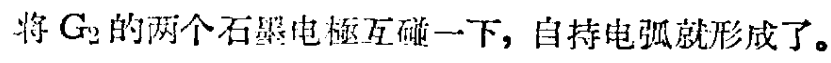

践们曾以含 $\mathrm{ZnO}$ 及 $\mathrm{CdO}$ 各 $0.1 \%$ 的 $\mathrm{SiO}_{2}$ 为对 潒，以数利条件进行多次摄㬐，以試驗这光源的再現 性。所用的电極弥統如 圈2所示, 加热电流用16 安培，激登放电用普通 引燃交流电弧以及 $\mathrm{L}_{1}$ 的 为 $0.5 \mathrm{mH}_{1} \mathrm{C}_{1}$ 为 20 及 $.50 \mu \mathrm{F}$ 的电容放电。用 这些条件都得到士12\% 公右的均方偏美（所用 和为 $\mathrm{Zn} \mathrm{3392.59/Cd}$ :3261.06)。应部指出，这 只是初步試駿的結果， 所用的工作条件不能說 是最好的; 扭且所用的

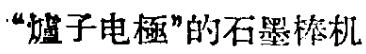
㭜性能很茬，粘构很不 均匀。这些都是能㨁接 影响再現性的因素。但

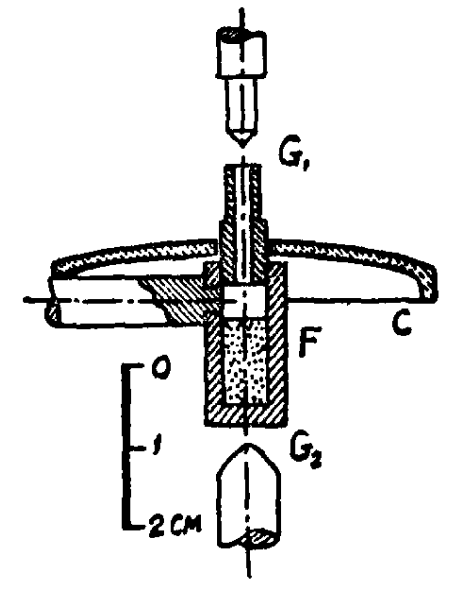

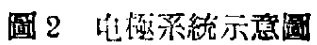
$\mathrm{G}_{1}$ 一激發比䋇， $\mathrm{G}_{2}$ 一加热 电弧，F一一装有試样的“㯰子

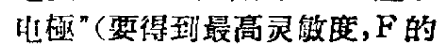
尺寸可以适当地增大, 以装盛较 多留試样)，C一坩佩盖，頂上 銘有小孔。
是，尽管如此，我倐所得到的再現性已綛比前迅文嗝 所报导的都好。

此外，我們还初步进行了一些利用不同的激策

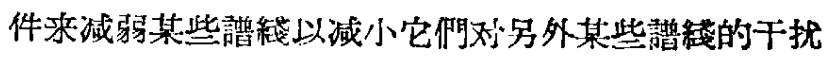
的試驗，这些試驗的結果都是很有希㕵的。

如果要得到更高的加热效䇣，可用直流电作为电 路中溞流部分的电源，抹以“庵子电極”为陽極。这时 在 $\mathrm{C}_{1}$ 的充电迴路中須加入一个较大的鉄心据流圈, 怙且 $R_{1}$ 不能太小。

目前秘們正对这种电路作进一步的的究和改进, 抹作备用它来进行某些非金屬元采分析的研究。

作者对这用化学研究所哭学周所长对本工作所給了的关

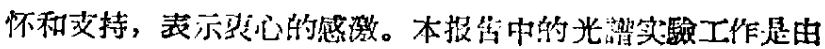

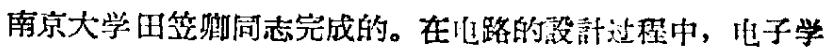
磔宛所楼弊明同诘提供了不少有益的意見。作者对田、楼两 同志表示感謝。

\section{黃 本立}

(中国科学院应用化学磔究所) 1957 年 4 月 19 日

[1] D. M. Shaw, O. I. Joensuu and L. H. Ahrens, Spectrochimica Acta, 4, 233(1950).

[2] D. M. Shaw, Geochim. et Cosmochim. Acta, 2, 118(1952).

[3] D. M. Shaw, 同.上, 2,185(1952).

[4] K. H. Wedepohl, 同. E, 3,93(1953).

[5] М. Л. Шиллинг, Зєвод. Лс.б., 22,447(1950).

[6] С. И. Абрамсон, 同. L., 14,1135(1948).

\section{二亚硒酸三軍鈵, $\mathrm{NaH}_{3}\left(\mathrm{SeO}_{3}\right)_{2}$ 的晶体結构}

\section{二亚硒酸三昰釣昆体乐掞 J. Janitzki 的工作[1]} 制得，其含硒量的实驗値和計算值各为 56.51 和 $56.42 \%$ 。晶体的 Laue 对称性为 $C_{2 h}$ 。晶体单科晶胞 的参数为

$$
\begin{aligned}
& a=5.90 \pm 0.02 \dot{A} \\
& b=4.82 \pm 0.02 \AA \\
& c=10.19 \pm 0.03 \dot{A} \\
& \beta=91^{\circ} 20^{\prime}
\end{aligned}
$$

晶胞中会有 $2\left\{\mathrm{NaH}_{3}\left(\mathrm{SeO}_{3}\right)_{2}\right\}$ 。泌度的許算值和实驗 值各为 3.224 和 $3.21 \pm 0.01$ 克/ 埋米3。根据系統消光, 昆体結构中存在着滑移面 $n$ 。最后根据全部原子的丟 数值, 晶体的空間群确定为 $C_{s}^{2}-P n_{0}$ 。

根据 Weissenberg 圖 hol 上各行射点的分布情 况, 可为結构在 $(010)$ 面上的投影引㑁一 $-a^{\prime}=5.94 \AA$, $b^{\prime}=5.90 \AA, \gamma^{\prime}=62^{\circ}$ 的假六方单位, 后孝的假平面群 